\title{
Instrumentos de Evaluación Psicológica en las Peritaciones de Guarda y Custodia de los Niños: Uso y Admisibilidad
}

\section{Psychological Assessment Tools in the Forensic Evaluation of Child Custody: Use and Admissibility}

\author{
Mila Arch-Marín y Adolfo Jarne-Esparcia \\ Universitat de Barcelona
}

\begin{abstract}
Resumen. El artículo presenta una revisión teórica de las dificultades y beneficios del uso de los instrumentos de evaluación psicológica que se aplican a los adultos en las evaluaciones periciales de guarda y custodia de los niños. Se comentan los datos disponibles sobre el uso de los tests psicológicos en estas evaluaciones a nivel internacional, pero con especial incidencia en la realidad española y una síntesis de las condiciones jurídicas para la admisibilidad de estas pruebas tanto en el entorno norteamericano como en España. Se realiza un análisis crítico de los instrumentos más utilizados en nuestro contexto en la exploración de los adultos ofreciendo consideraciones de utilidad para los técnicos que realizan estas intervenciones en relación al uso de los instrumentos de evaluación.

Palabras clave: evaluación pericial, guarda y custodia, instrumentos de evaluación, admisibilidad.
\end{abstract}

\begin{abstract}
The article presents a theoretical review of the difficulties and benefits of using psychological assessment tools directed toward adults in forensic evaluations of child custody. We discussed: (1) the international information available about the use of psychological tests in these assessments, with a special focus on Spanish reality and (2) a summary of the legal conditions for the admissibility of these tests in the North-American and Spanish contexts. A critical analysis of the more used tools is performed in our context of the assessment in adults, providing useful considerations for technicians in relation to the application of such tools in child custody evaluations.

Keywords: forensic evaluation, child custody, psychological tests, admissibility.
\end{abstract}

La consolidación de las funciones del psicólogo forense en el ámbito de familia y más concretamente, en la evaluación pericial de la guarda y custodia de los niños, es un hecho constatado, tanto en el contexto norteamericano (Erickson, Lilienfield y Vitacco, 2007) como en España (Arch, 2008). A pesar de que se han realizado críticas sobre la poca utilidad de las eva-

La correspondencia sobre este artículo deberá enviarse a la primera autora al Departament de Personalitat, Avaluació i Tractament Psicològic. Facultat de Psicologia. Universitat de Barcelona. Passeig de la Vall d’Hebrón, 171.08035 Barcelona (España).E-mail: march@ub.edu luaciones de salud mental en el ámbito de los tribunales de familia (e.g.: Tippins y Wittmann, 2005), la valoración general de estas intervenciones es positiva tal como demuestra la frecuencia de su solicitud o admisión por los tribunales.

Son diversas las cuestiones sobre las que se consulta a los psicólogos que actúan en este ámbito (adopciones, filiación...), sin embargo, el principal requerimiento de los jueces y magistrados suele dirigirse fundamentalmente a encomendarle que dictamine sobre la idoneidad 
de que la guarda y custodia de los hijos del matrimonio - o pareja de hecho - en litigio le sea concedido a uno u otro de los cónyuges o a ambos de forma compartida. Las estimaciones en este sentido indican que, en España, al menos el 80 por 100 de demandas de periciales psicológicas en los juzgados de familia corresponde a asuntos matrimoniales y de ellos una proporción variable pero no inferior a la mitad, son dictámenes de custodia (Ramírez, 2003).

La evaluación pericial en estos casos entraña una enorme dificultad (Otto, Edens y Barcus, 2000) ya que el psicólogo forense se enfrenta a una intervención que requiere la exploración de diversas personas $\mathrm{y}$, por tanto, de un gran número de variables. Asimismo, se ha resaltado la dificultad añadida que entraña esta labor por cuanto supone una de las más estresantes para los profesionales a causa de los altos niveles de emotividad y acritud que acostumbran a encontrarse asociados a los procesos de ruptura familiar (Kirkland y Kirkland, 2001). La complejidad de la intervención se evidencia también en los resultados ofrecidos por los autores que han explorado la frecuencia de quejas recibidas en las Comisiones Deontológicas de las asociaciones y colegios profesionales y que muestran que un alto número de las mismas corresponden a intervenciones periciales $\mathrm{y}$, concretamente, en su mayoría referidas a evaluaciones realizadas en el ámbito de familia (Cayuela, Jarne y Molina, 2004; Montgomery, Cupit y Wimberly, 1999).

Aunque desde nuestra disciplina se han propuesto diferentes modelos para la evaluación de la guarda y custodia (e.g.: Brickiln, 1995; Collier, 1996; Fariña y Arce, 2006; Ramírez, 1997), los datos empíricos que muestran las prácticas reales de los profesionales son aun limitados (Ackerman y Ackerman, 1997; Arch, 2008; Bow y Quinell, 2001; Keilin y Bloom, 1986; Lafortune y Carpenter, 1998).

Los resultados provenientes de nuestro país (para una revisión ver: Arch, 2008), nos indican que la metodología de evaluación (procedimientos y técnicas) de los psicólogos forenses españoles ofrece un perfil básico que se adecua a las recomendaciones genéricas de las guías especializadas en la materia (AACAP, 1997; AFCC, 2006; APA, 1994) y, aunque con matices, a las apreciadas en sus investigaciones por los autores norteamericanos (Ackerman y Ackerman, 1997; Bow y Quinnell, 2001, 2002; Lafortune y Carpenter, 1998; Keilin y Bloom, 1986). En este diseño metodológico seguido por los profesionales, destaca la frecuencia de uso de test psicológicos en la exploración de los adultos como parte de la evaluación; posiblemente potenciado en este entorno profesional, tal como indica Brodzinsky (1993), porque su uso por parte de los peritos resulta muy atrayente para los juristas que consideran que la evaluación cobra objetividad científica si se encuentra apoyada por los resultados de estos instrumentos. Sin embargo, como destacaron Bow y Quinell (2001) y Lafortune y Carpenter (1998), a pesar de la evidencia de frecuencia de uso de los test psicológicos en las periciales de custodia, los profesionales les otorgan un valor moderado de importancia para su valoración frente a otras técnicas como las entrevistas individuales con cada progenitor y con el menor o la observación de la interacción parento-filial.

A pesar de la evidencia de frecuencia de la intervención del psicólogo forense en las evaluaciones de custodia, la utilidad de estas periciales ha sido criticada tanto por juristas como por profesionales de salud mental (Bow, Gould, Flens y Greenhut, 2006). En relación con ello, la bibliografía científica muestra un gran debate sobre la adecuación del uso de tests psicológicos en la evaluación de custodia (Bricklin 1992, 1999; Brodzinsky, 1993, Grisso, 1986, 1990; Quinell y Bow, 2001; Ramírez, 2006). En este contexto, el objetivo del presente trabajo fue realizar una revisión crítica de los instrumentos de evaluación psicológica aplicados a los adultos en valoraciones periciales de custodia y de las normas jurídicas que guían la admisibilidad de los mismos por parte de los tribunales; ofreciendo pautas concretas derivadas de ambos aspectos que pueden contribuir a facilitar la labor de los psicólogos que intervienen en procedimientos de familia. 


\section{El debate entorno al uso de test en la evaluación de la guarda y custodia}

En la literatura científica se delimitan diversos factores que cuestionan de forma importante la práctica habitual de utilizar instrumentos psicológicos (psicométricos y/o proyectivos) en las evaluaciones de custodia. Entre estos diversificados posicionamientos contrarios aparece en primer lugar una crítica que tiene relación con la propia validez del uso en entorno pericial de instrumentos que fueron diseñados para el ámbito clínico. Este hecho fue ya remarcado por Grisso en 1986, llamándose la atención sobre esta cuestión de manera periódica (Bricklin, 1992, 1999; Brodzinsky, 1993). El argumento básico es que los aspectos que interesan ante los tribunales de familia como son la capacidad parental, la recomendación del tipo de guarda y custodia, los vínculos familiares, etc. no son recogidos por el tipo de instrumental psicológico que los profesionales utilizan, que provienen generalmente del campo clínico o de la evaluación de la personalidad y, por lo tanto, sus resultados no resultan pertinentes para decidir sobre las cuestiones sobre las que pregunta el tribunal. No obstante, se acepta que existe algún nivel de relación entre la presencia de psicopatología en los padres o los rasgos de personalidad y las capacidades parentales o la decisión sobre la custodia. Así, algunos autores (Taylor y cols, 1991; Erickson, Lilienfeld y Vitacco, 2007) han señalado que la presencia de psicopatología activa en un padre aumenta la dificultad para actuar como cuidador e incluso los riesgos para la seguridad del menor. Respecto a la personalidad, tal y como ya indicó Ramírez (2006), en nuestro país ha recibido mucho énfasis en las evaluaciones de custodia, especialmente en los momentos en que la evaluación se regía por criterios negativos tratando de contrastar la presunta incapacidad de uno de los progenitores. El mayor argumento contra ello es que es prácticamente imposible establecer un perfil de personalidad "bueno" frente a uno "malo". El mismo concepto de personalidad contradice esta idea. $\mathrm{Si}$ a ello se añade que no existe relación directa entre rasgos de personalidad primarios y capacidades parentales (no es mejor padre/madre el introvertido sobre el extrovertido y viceversa), la exploración de personalidad en contexto de familia perdería en gran medida su sentido. Paralelamente es necesario recordar que la presencia de ciertos indicadores de desajuste en el momento de evaluación, pueden responder a aspectos circunstanciales contingentes con la situación de crisis y no llegar a conformar una entidad realmente psicopatológica o bien, el momento de crisis puede exacerbar estados que se encontraban compensados o en estado premórbido (Ibáñez y Ávila, 1990).

\section{El uso de test psicológicos en las evaluaciones periciales de guarda $y$ custodia}

Como indicábamos, el uso de test psicológicos en las evaluaciones de custodia es generalizado. En relación a la exploración de los adultos, los datos disponibles sobre su uso real por parte de los profesionales especializados en evaluaciones de guarda y custodia se encuentran detallados en la tabla 1. Como puede apreciarse, los datos españoles reflejan, en primer lugar, la ausencia de test específicos diseñados $\mathrm{y}$ validados para el entorno forense, posiblemente por este motivo, los psicólogos forenses recurren al uso de instrumentos procedentes del psicodiagnóstico tradicional.

El instrumento psicométrico más empleado, por los psicólogos forenses españoles expertos en el ámbito de familia, es el cuestionario 16 PF-5 (Cattell y Catell, 1995), ello podría indicar la convicción de los técnicos españoles de que los rasgos de personalidad de los progenitores influyen en sus habilidades parentales; aspecto evidenciado también en el estudio de Bow, Gould, Flens y Greenhut (2006), apreciando que la exploración de los rasgos de personalidad eran considerados por los peritos como un aspecto de especial importancia en las evaluaciones de custodia. Sin embargo, como puede apreciarse en la tabla 1, resulta bastante inusual en este tipo de evaluaciones en el contexto nor- 


\begin{tabular}{lcccc}
\multicolumn{5}{c}{ Tabla 1: Distribución por autores de los porcentajes observados de uso de diversos instrumentos psicológicos en evaluaciones de guarda y custodia } \\
\hline Tests & Arch (2008) & Quinell y Bow (2001) & Ackerman y Ackerman (1997) & Keilin y Bloom(1986) \\
\hline 16PF-5 & 69 & 9 & 8 & 6 \\
MMPI2 & 60 & 94 & 32 & 71 \\
MCMI-II/III & 60 & 52 & 6 & - \\
HTP & 19 & 10 & - & - \\
STAI & 12 & - & - & - \\
EPQ-R & 10 & - & 48 & - \\
ROSCHACH & 10 & - & - & - \\
SCL-90 & 10 & 47 & 43 & 29 \\
WAIS & 8 & 26 & 22 & 12 \\
Frases incompletas & - & 24 & 29 & 38 \\
TAT & - & &
\end{tabular}

teamericano, donde los estudios revisados (Ackerman y Ackerman, 1997; Bow y Quinell, 2001; Keilin y Bloom, 1986) indican un uso claramente minoritario de este instrumento.

En las muestras norteamericanas el instrumento más utilizado en la valoración del adulto es el MMPI-2; el 94\% de los profesionales que conformaron la muestra del estudio de Bow y Quinell (2001) lo usaban en sus evaluaciones de custodia. Como es sabido, este cuestionario mide la personalidad anormal y, por tanto, contribuye a la detección de patología en el adulto que pudiese interferir en su función parental. Los datos españoles sitúan el uso del MMPI-2 en segunda posición (60\% de la muestra) pero al mismo nivel de uso que el cuestionario MCMI-II (Millon, 2005) que es aplicado en España con una frecuencia ligeramente superior a la recogida de las muestras norteamericanas.

En relación a las pruebas proyectivas se aprecia que, aunque en menor medida, los profesionales, tanto españoles como norteamericanos emplean el HTP y el Rorschach, este último, con una notable presencia en el contexto estadounidense donde casi la mitad de las muestras que conforman los estudios disponibles informan utilizarlo en las evaluaciones de guarda y custodia. Asimismo, también utilizan con cierta frecuencia otros instrumentos proyectivos, concretamente, las frases incompletas y el Test de Apercepción Temática.

En nuestro país aunque ciertamente en porcentajes muy limitados los psicólogos forenses informan el uso de cuestionarios como el STAI
(Spielberger, Gorsuch, y Lushene, 1970), el EPQ-R (Eysenck y Eysenck, 1997b) y el SCL90 (Derogatis, 1977), cuyo uso en el contexto norteamericano es inexistente.

Finalmente, apreciamos discrepancia entre la frecuencia de uso del WAIS en el entorno norteamericano y el español, ya que mientras en nuestro contexto su uso se encuentra claramente limitado (8\%), en las muestras americanas el porcentaje se aproxima a la mitad de la muestra, siendo por tanto, mucho más frecuente su aplicación.

No se revisan en el presente artículo por la imposibilidad de comparación con nuestro contexto el extendido uso en Estados Unidos de instrumentos forenses diseñados de forma específica para la valoración de la custodia. Sin embargo, creemos importante apuntar que a pesar de la clara necesidad de disponer de instrumentos forenses que nadie duda, los intentos realizados hasta el momento en Norteamérica, donde son ampliamente aplicados por los técnicos (Ackerman y Ackerman, 1997) no están exentos de criticas que ponen en duda su validez de constructo (Heinze y Grisso,1996).

\section{El MMPI-2 y las evaluaciones de custodia}

Mucho se ha debatido entorno al uso de este cuestionario en las evaluaciones de custodia. Básicamente los diferentes autores que han tratado esta cuestión centran el interés en dos grandes aspectos: la frecuencia de perfiles defensi- 
vos y su interpretación en este contexto y la interpretación adecuada en las evaluaciones de custodia de elevaciones en determinadas escalas clínicas.

La producción de estilos de respuesta defensivos en el perfil del MMPI-2 es un hecho constatado por los profesionales respecto a los adultos explorados en una evaluación de guarda y custodia (Bagby, Nicholson, Buis, Radovanovic y Fidler, 1999; Ogloff, 1995). Una defensividad que se define como la minimización o negación de síntomas que puede producirse en presencia de una recompensa externa (Vitacco y Rogers, 2006). Algunos autores (e.g. Erickson, Lilienfield y Vitacco, 2007) indican que estos perfiles se obtendrían en la mayoría de los casos evaluados, superando de forma amplia la frecuencia observada en muestras de pacientes psiquiátricos hospitalizados (Posthuma y Harper, 1998; Siegel, 1996). Cabe recordar que los estilos de respuesta altamente defensivos tienen la capacidad de anular la totalidad de resultados de la prueba y que, aun suponiendo que los resultados sean suficientemente interpretables, la defensividad puede alterar los mismos en las escalas clínicas requiriendo la suma cautela del técnico en las interpretaciones. Además de ello, algunos autores (e.g. Hartman-Crouch, 2000) han alertado sobre la práctica de algunos profesionales que interpretarían el perfil obtenido a pesar de que las escalas de validez indicasen la invalidez del mismo y, por tanto, sin garantías de fiabilidad en las valoraciones extraídas por este medio.

La segunda cuestión relacionada con el uso del MMPI-2 en las evaluaciones de custodia la encontramos en la investigación centrada en su utilidad para detectar psicopatología en el contexto de estas evaluaciones. En este sentido, se ha señalado una frecuente elevación de las escalas de desviación psicopática y paranoia (Bathurst, Gottfried y Gottfried, 1997; Hartman-Crouch, 2000). Sin embargo, la interpretación de estas elevaciones, en particular la de desviación psicopática, debe ser prudente dado que puede venir provocada por el mismo contexto de la intervención, es decir, en sujetos que están experimentando importante conflictividad conyugal (Otto y Collins, 1995).

\section{EI MCMI-II /MCMI-III y las evaluaciones de custodia}

Tal y como indican (Quinell y Bow, 2001) el uso de las escalas MCMI en entorno forense no esta exento de controversia, especialmente en la más reciente edición de la prueba (MCMI-III). Concretamente, Rogers, Salekin y Sewell (1999) indican que el MCMI-III carece de validez de criterio para los trastornos del eje II y, como prueba psicológica, no cumple las normas Daubert (1993). En el articulo critico escrito por Erad (2007) respecto al publicado por Erickson Lilienfield y Vitacco (2007), el autor señalaba numerosos aspectos que condicionaban el uso del instrumento, entre otros, el bajo poder predictivo de los resultados y la existencia de sesgo de género en la estandarización del instrumento.

De forma similar que en el caso del MMPI-2, diversos autores (Lampel, 1999; McCann, Flens, Campagna,Collman, Lazzaro y Connor, 2001) han alertado sobre la alta posibilidad de obtener perfiles defensivos en la prueba cuando se trata de una evaluación de guarda y custodia de los hijos, en especial se aprecian elevaciones en la escala "Y" (Conveniencia). Asimismo, se observa una tendencia a la elevación subclínica de las escalas 4 (histriónico), 5 (narcisista) y 7 (compulsivo).

A pesar de estas criticas autores como Stredny, Archer y Mason (2006) han señalado la utilidad del cuestionario como medio de exploración de los problemas emocionales y de personalidad que puedan presentar los progenitores $\mathrm{y}$ afectar de forma negativa a su funcionamiento parental.

\section{Las escalas Wechsler}

Como hemos visto, en el contexto norteamericano la aplicación de las escalas Wechsler (Wechlser, 1955) a los adultos implicados en 
una valoración de custodia es frecuente. Sin embargo, resulta evidente que la estimación de la capacidad intelectual no informará al evaluador sobre el conocimiento que un progenitor tiene sobre su hijo, la relación que mantienen o el conocimiento y comprensión sobre el ajuste emocional del hijo (Otto, Edens y Barcus, 2000). Tal y como señalan Baerger, GalatzerLevy, Gould y Nye (2002) salvando la excepción de la presunción de existencia de un grave deterioro cognitivo que imposibilitase el cuidado adecuado de los niños, no se encuentra empíricamente establecida la relación entre el funcionamiento intelectual y la capacidad de crianza de los hijos. Por tanto, es poco probable que la información sobre la capacidad cognitiva contribuya a la valoración de habilidades parentales. De hecho, los citados autores concluyen que estas pruebas no son pertinentes para la evaluación de la idoneidad para la ostentación de la guarda y custodia, porque la información que aportan es irrelevante. Asimismo, señalan el riesgo y posible perjuicio derivado de que los tribunales puedan malinterpretar los datos provenientes de valoraciones cognitivas de los progenitores estimando que el progenitor que obtenga una mejor puntuación ("más inteligente") pueda constituir un "mejor padre" y, consecuentemente un mejor cuidador del hijo.

\section{Técnicas proyectivas y evaluaciones de custodia}

Aunque las técnicas proyectivas gozaron de un gran reconocimiento durante más de cuatro décadas, considerándose durante ese tiempo como las más importantes, a partir de los años sesenta han suscitado numerosas críticas entre los expertos en evaluación psicológica, derivadas, entre otros factores, del escaso soporte empírico constatado a través de la investigación (Marquez, Vizcarro y Fernández-Ballesteros, 2004).

Cabe recordar, asimismo, que el uso de pruebas proyectivas en entorno forense ha recibido duras criticas por parte de los expertos (Grisso,
1990; Lilienfield, Wood, y Garb, 2000; Medoff, 2003); aunque se haya reconocido cierta utilidad por su calidad de "test enmascarados", difíciles de falsear, ya que el sujeto no presentaría conciencia de la relación entre sus respuestas y su mundo interno (Anastasi, 1998; Marquez, Vizcarro y Fernández-Ballesteros, 2004). Asimismo, Rodríguez Sutil (1999, 2007) ha señalado la bondad de los sistemas de corrección y puntuación que se han desarrollado entorno a algunas pruebas proyectivas y concretamente al propuesto por Exner (1974, 1978, 1986) en relación al test de Rorschach.

Sin embargo, y respecto al uso concreto en evaluaciones de custodia de pruebas proyectivas basadas en el dibujo, la literatura científica señala con claridad que su fiabilidad y validez puede ser débil o inexistente (Baerger, GalatzerLevy, Gould y Nye, 2002; Erickson y cols., 2007; Hynan, 2003; Lally, 2001). Como hemos visto, el uso del Rorscharch se encuentra notoriamente extendido en las evaluaciones de custodia en el contexto norteamericano (no así en el español). De hecho, su uso sistemático aparece recogido en el protocolo de una de las pruebas diseñadas específicamente para la evaluación de custodia en el contexto norteamericano creada por Ackerman y Schoendorf (1992), el ASPECT.

Esta situación nos sugiere que las pruebas proyectivas y, en particular, el uso del test de Rorscharch, cuentan con un buen nivel de confianza de los técnicos norteamericanos especialistas en estas evaluaciones a pesar de las criticas que se han vertido sobre dichos instrumentos en el entorno forense.

\section{Recomendaciones y consideraciones sobre el uso de instrumentos en la evaluación forense}

El dictamen emitido por el perito en relación a la materia sobre la que se le ha interrogado es un medio de prueba dentro de un proceso judicial, que será ponderada y valorada por el juez en su propia toma de decisión para dictar la sen- 
tencia, siendo responsabilidad de este último el peso que otorgara a la valoración del perito (Ortuño, 1998). Sin embargo, algunos autores han resaltado el peso real constatado que tienen los informes periciales en las decisiones judiciales. Granados (1990) hace notar esta circunstancia subrayando además la incidencia que supondrá esta valoración sobre la vida afectiva, económica, etc. de las personas, en la medida en que la sentencia recoja las opiniones de la pericia. En este sentido nos advierten también Aguilera y Manrique (2003) al ofrecernos el resultado de un estudio realizado en España al señalar como conclusión más importante " $e l$ papel decisivo, que según los jueces, tiene el informe psicológico para valorar los aspectos centrales sobre los que centra la pericia psicológica", indicándonos que, en su estudio, "la mayor parte de los jueces, responden que sólo en algunas ocasiones, toman decisiones diferentes a las recomendadas en los informes". Mucho mas allá llega Montero (2001), desde el entorno jurídico, al indicar que en algunas ocasiones el contenido de la Sentencia emitida por el juez es una simple remisión a lo expresado por el perito en el informe.

Esta constatación del alto peso que supone la pericial en el proceso de toma de decisión del juez, constituye un aspecto fundamental a considerar por el profesional que se enfrenta a la realización de una evaluación pericial $\mathrm{y}$, por ello, tal y como indican las directrices especializadas (Specialty guidelines for forensic psychologists, 1991) la intervención debe realizarse bajo los más altos estándares técnicos y éticos.

\section{La valoración judicial de la prueba pericial}

En EE.UU., país con mucha más tradición en estas intervenciones periciales, el juzgador dispone de criterios explícitos para la valoración de los dictamenes periciales. En el periodo comprendido entre los años 1923 a 1993 los tribunales norteamericanos se guiaban por la norma Frye ( Frye v. United States, 293F. 1013, 1923) a la hora de valorar la admisibilidad de un testimonio experto; según este estándar, las evidencias presentadas por el técnico en su valoración debía estar "suficientemente establecida para haber conseguido la aceptación general en el campo concreto al que pertenece"; por tanto, los principios e instrumentos utilizados debían contar con la "aceptación general" de su área científica.

A raíz de la decisión adoptada por el Tribunal Supremo de EE.UU. en el caso "Daubert contra Merrell dow Pharmaceuticals" (1993), se produce una ampliación del criterio para la admisibilidad del peritaje científico, concretamente, en relación a la fiabilidad de la metodología seguida por el técnico; el juez deberá determinar: a) si la teoría o técnica subyacente ha sido probada, b) si la teoría o técnica ha sido objeto de revisión por pares y ha sido publicada, c) si los instrumentos utilizados tienen una tasa de error conocida y d) si han conseguido aceptación general de su ámbito científico (Meyer, 2006; Bow, Gould, Flens y Greenhut, 2006). De esta forma, el tribunal puede valorar que lo expuesto por el técnico en su pericia no responde a una mera especulación o a vagas generalidades pseudocientificas sino que se encuentra sostenido por métodos sólidos, fiables y pertinentes que son aplicables en el caso concreto evaluado. La norma Daubert se vio complementada a través de dos Sentencias posteriores del Tribunal Supremo estadounidense (General Electric Company v. Joiner, 1999; Kumho Tire Co. v. Carmichael, 1999), subrayando la necesidad de valorar la fiabilidad del método utilizado como base de las valoraciones del perito y ampliando la aplicación de la norma a áreas del conocimiento más técnicas. Según refieren Bow, Gould, Flens y Greenhut (2006), con estas aclaraciones no podía ponerse en duda que la norma afecta también a los psicólogos, que deben ir más allá de la comúnmente usada regla de "aceptación general" y centrarse en factores más científicos como la fiabilidad, validez y relevancia de los instrumentos y teorías; naturalmente, el testimonio de los expertos respecto a la competencia para ostentar la guarda y cus- 
todia de los hijos no es una excepción y, por tanto, deben cumplir las normas de admisibilidad (Baerger, Galatzer-Levy, Gould y Nye, 2002).

Con anterioridad a la norma Daubert, algunos autores (e.g. Hailbrun, 1992) ya habían sugerido la conveniencia de usar criterios más estrictos en la elección de las pruebas psicológicas a aplicar en las peritaciones, facilitando una serie de puntos básicos a considerar por el psicólogo (ver tabla 2); el seguimiento de estas directrices ayuda al profesional a mantener un sistema afín a los criterios explicitados en la norma Daubert. vinculado a o determinado por los peritos para adoptar su propia decisión. La dificultad de este criterio establecido en nuestro país, estriba en la consideración objetiva de que elementos conforman "la sana critica" habiéndose asemejado desde el entorno jurídico, entre otros, a conceptos como "el razonar humano", "las más elementales directrices de la lógica humana", "normas racionales " o "sentido común" (Zubiri, 2006). Este autor, en función de lo establecido por la regulación jurídica (Articulo 478 de la LECrim), sugiere los puntos fundamentales que deben reflejarse en los informes periciales para

Tabla 2. Directrices a contemplar en la selección de técnicas e instrumentos. (Adaptado, Hailbrun, 1992)

Los instrumentos utilizados deben estar comercializados y por tanto haber pasado un proceso de revisión sólido, acompañados de un manual que describa su desarrollo, las propiedades psicométricas (fiabilidad y validez), normas de aplicación, etc.

Constatar que están disponibles los datos de normalización del instrumento.

Asegurarse que el nivel de fiabilidad de la prueba esta adecuadamente demostrado. Extremar la prudencia en el uso de instrumentos con estimaciones de fiabilidad inferiores a 0.80 .

Además de la fiabilidad, cabe constatar la validez de la prueba, consultando a tal efecto los datos que deben constar en el manual.

Asegurarse que la prueba utilizada es pertinente para la cuestión que se formula desde los juristas. Ello debería estar establecido, siempre que sea posible, por la validación de investigaciones publicadas en revistas con proceso de revisión anónima por pares.

El examinador deberá considerar las diferencias existentes entre los fines para los que se desarrollo la prueba originalmente y los correspondientes a lo que se está evaluando en entorno forense.

Seguir de forma estricta los requisitos para la aplicación de la prueba, incluyendo la adecuada formación y experiencia de quien la administra.

La aceptación generalizada de la norma Daubert propició que los investigadores en la materia centrase su interés en divulgar aspectos que centrasen aun en mayor medida la atención de los técnicos en la necesidad de ser más críticos y cuidadosos en la selección de pruebas psicológicas para las evaluaciones de guarda y custodia (e.g.: Marlowe, 1995; Otto,Edens y Barcus, 2001).

En nuestro país, el criterio de valoración de la prueba pericial por parte del tribunal queda recogido en el Articulo 348 de la NLEC, donde se indica que "el tribunal valorará los dictámenes periciales según las reglas de la sana critica", según refiere Ledesma (2006), ello supone, en primer lugar, rechazar la opción de que el juzgador deba someterse a unas reglas concretas a través de las que otorgue un determinado valor a la pericia; en segundo lugar, la libre apreciación de la prueba evita que el juzgador se vea la adecuada valoración judicial y que se encuentran detallados en la tabla 3.

Zuribi (2006), en referencia a lo sugerido por Cobo (2000) respecto a la pericial forense en casos de violencia de género, también recoge las indicaciones ofrecidas para la valoración de una prueba biomédica (ver tabla 4), como puede apreciarse, estas directrices, resultan conceptualmente muy próximas a los criterios anglosajones descritos anteriormente.

\section{Implicaciones para la práctica}

La conclusión principal de esta revisión es que la práctica habitual de la evaluación pericial de los adultos en un proceso judicial de familia incluye la administración de test y pruebas psicológicas provenientes de otro contexto, mayoritariamente de la clínica. Aunque se admite que 
Tabla 3. Puntos fundamentales que debe incorporar el informe pericial. (Adaptado. Zubiri,2006)

Aspecto necesario
Hacer constar los elementos efectivos de los que parte para la realiza-
ción de la pericial. Reflejando los hechos y fuentes de conocimiento
en que se basa a fin de facilitar su contrastación.
Informar de las operaciones (reglas de la ciencia) que el técnico ha
llevado a cabo para la elaboración de su dictamen. Teniendo en cuen-
ta que:
- El status científico de una teoría viene determinado por su someti-
miento a procesos de refutabilidad y control.
- Las afirmaciones que constituyen una explicación científica deben
ser susceptibles de una verificación empírica.

Valoración del juzgador

En caso de discrepancia el juez podrá determinar si ésta proviene del método de valoración utilizado, de las apreciaciones que el perito realiza como experto o de que parte de unos hechos diferentes a los valorados por otro técnico o de los contrastables por otros medios.

El juzgador puede constatar que el perito haya actuado conforme al método científico y valorar la fiabilidad de su dictamen.

Tabla 4. Directrices para valoraciones con menor carga de evidencia directa. (Zuribi, 2006 en referencia a Cobo, 2000)

La teoría científica subyacente es válida.

La técnica que se aplica a la teoría es válida.

La técnica se aplica adecuadamente a la situación concreta.

La prueba aplicada debe ser aceptada como de valor suficiente por la comunidad científica en general y estar publicada habiendo seguido un proceso de revisión por pares.

Se deben poder comprobar los métodos usados.

Debe existir una determinación de la frecuencia de error en sus resultados.

Debe existir la posibilidad de una revisión externa por otros especialistas.

El experto debe disponer de una cualificación adecuada.

Los resultados deben poder ser replicados, dentro de lo posible.

Los criterios y términos usados deben ser simples, de fácil comprensión para los tribunales y jurados.

en ocasiones y aspectos puntuales estos instrumentos pueden resultar de utilidad a los peritos, las críticas a su uso generalizado son muy importantes, especialmente, en el caso de las pruebas proyectivas.

Aunque la regulación española muestra un criterio ambiguo en cuanto a la admisibilidad del dictamen pericial, como ha podido apreciarse, los criterios explicitados por juristas de nuestro país resultan muy próximos a lo establecido en el contexto norteamericano a través de la norma Daubert. Ello evidencia que a fin de dotar de mayor calidad las intervenciones periciales en el ámbito de familia, resulte muy importante que los técnicos que las realizan constaten el cumplimiento y adaptación a las citadas normas, de las técnicas e instrumentos usados en su valoración.

\section{Referencias}

Ackerman, M.J. y Ackerman, M.C. (1997). Custody evaluation practices: a survey of experienced professionals revised. Profesional psychology: research and practice, 2.

Aguilera, G. y Zaldívar, F. (2003). Opinión de los jueces (derecho penal y de familia) sobre el informe psicológico forense. Anuario de psicología jurídica, 13.

American Psychological Association (1994). Guidelines for child custody evaluations in divorce proceedins. American Psychology, 49, 677680 .

Anastasi, A. (1998). Test psicológicos. México: Aguilar.

Arch, M. (2008). La intervención de los psicólogos forenses en las evaluaciones de la guarda y cus- 
todia de los niños (Tesis doctoral, Facultad de Psicologia, 2008). Disponible en http://www. tdx.cat/TDX-1103108-114532

Association of Family and Conciliation Courts (2006). Model estandards of practice for child custody evaluation. Recuperado el 30/03/2009 de: http://www. afccnet.org/pdfs/Model\%20Stds\%20Child\%20Cust ody\%20Eval\%20Sept\%202006.pdf

Bathurst, K, Gottfried, A.W. y Gottfried, A.E. (1997). Normative data for the MMPI-2 in child custody litigation. Psychological Assessment, 9, 205-211.

Bow, J.N., Gould, J.W., Flens, J. R. y Greenhut, D. (2006). Testing in child custody evaluations. Selection, usage and Daubert admissibility: A Survey of Psychologists. Journal of Forensic Psychology Practice, 6, 17-38.

Bow, J. N. y Quinnell, F. A. (2001). Psychologists current practices and procedures in child custody evaluations: Five years after APA guidelines, Professional Psychology: Research and Practice, 32, 261-268.

Bricklin, B. (1992). Data-based tests in child custody evaluations. American Journal of Family Therapy, 20, 254-265.

Bricklin, B. (1995b).The custody evaluation handbook: Research based solutions and applications. New York: Brunner-Mazel.

Bricklin, B. (1999). The contributions of psychological tests to child custody evaluations. En R. M. Galatzer-Levy and L. Kraus (Eds.), The scientific basis of child custody decisions (pp. 120-156). New York: John Wiley.

Brodzinsky, D. M. (1993). On the use and misuse of psychological testing in child custodyevaluations. Professional Psychology: Research and Practice, 24, 213-219.

Catell, R.B., Catell, A.K.S. y Catell, H.E.P. (1995). 16 PF-5 Cuestionario de personalidad para adultos. Madrid: TEA Ediciones, S.A.

Cayuela, R., Jarne, A., Molina, A. (2004). Estudio descriptivo de las denuncias atendidas por la comisión deontológica del colegio oficial de psicólogos de Catalunya en relación con las intervenciones periciales. Psicología Jurídica. Psicología y Ley, 1. Santiago de Compostela: Xunta de Galicia.
Collier, H.L. (1996). The analysis of family dynamics in child custody evaluations. In G. Davies, S. Lloyd-Bostock, M. McMurran y C. Wilson (Eds.). Psychology, law and criminal justice (pp. 231-239) Berlin: De Gruyter.

Committe on Ethical Guidelines for Forensic Psychologists. (1991). Specialty guidelines for forensic psychologists. Law and Human Behavior, 15, 655-665.

Derogatis, L. R.(1977). SCL-90. Adminstration, scoring and procedures.Manual. Baltimore: Clinical Psychometric Research.

Emery, R. E. (1999b). Postdivorce family life for children. En Thompson, R.A. y Amato, P.R. (Eds.), The postdivorce family: Children, parenting, and society. CA: Sage: Thousand Oaks.

Erard, R.E. (2007). picking cherries with blinders on: a comment on erickson et al. (2007) regarding the use of tests in family court. family court review, 45, 175-184.

Erickson, S.K., Lilienfeld, S.O. y Vitacco, M.J. (2007). Psychological testing and child custody evaluations in family court: a dialogue. a critical examination of the suitability and limitations of psychological tests in family court. Family court review, 45, 157-174.

Exner, J.E. (1974). The Rorschach; A Comprehensive System: Vol. I. Nueva York: John Wiley y Sons.

Exner, J.E. (1978). The Rorschach; A Comprehensive System: Vol. II. Nueva York: John Wiley y Sons.

Exner, J.E. (1986). Some Rorschach data comparing schizophrenics with borderline and schizotipal personality disorders. Journal of Personality Assessment, 50, 455-471.

Eysenck, H.J. y Eysenck, S.B.G. (1997b). Cuestionario Revisado de Personalidad de Eysenck (EPQ-R). Manual. Madrid: TEA.

Fariña, F. y Arce, R. (2006). El papel del psicólogo en casos de separación o divorcio. En J.C. Sierra, C. Jiménez y G. Buela-Casal (Eds.), Psicología forense: Manual de técnicas y aplicaciones (pp. 246- 271). Madrid: Biblioteca Nueva.

Frye v. United States, 293F. 1013 (1923).

Gardner, R.A. (1989). Family evaluation in child custody mediation, arbitration and litigation. Cresskill, NJ: Creative Therapeutics. 
General Electric Co. v. Joiner, 522 U.S. 136 (1997).

Granados, F. (1991). Etica y Técnica de la intervención psicológica en los juzgados de familia. Tesis doctoral. Universidad Complutense de Madrid.

Grisso, T. (1986). Evaluating competencies: Forensic assessments and instruments. New York: Plenum.

Grisso, T. (1990). Evolving guidelines for divorce/ custody evaluations. Family y Conciliation Courts Review, 28, 35-41.

Hartman-Crouch, T. S. (2000). The Minnesota Multiphasic Personality Inventory-2 in the context of child custody litigation.(Doctoral Dissertation, California School of Professional Psychology, 2000). Dissertation Abstracts, 61, 2762.

Hathaway, S. R. y McKinley, J. C. (1999). MMPI-2. Inventario Multifásico de Personalidad de Minnesota-2. Madrid: TEA Ediciones, S.A.

Heilbrun, K. (1992). The role of psychological testing in forensic assessment. Law and Human Behavior, 16, 257-272.

Heinze, M. C. y Grisso, T. (1996). Review of instruments assessing parenting competencies used in child custody evaluations. Behavioral Sciences y the Law, 14, 293-313.

Hynan, D.J. (2003). Parent- child observations in custody evaluations. Family court review, 41.

Ibáñez,V. y Ávila, A. (1990). Psicología forense y responsabilidad legal. En A. Garzón, Psicología Judicial. Valencia: Promolibro.

Karras, D. y Berry, K. (1985). Custody evaluations: a critical review. Professional Psychology, 16, 76-85.

Keilin.W.G. y Bloom, L.J. (1986). Child Custody Evaluation Practices: A survey of experienced professionals.Professional Psychology: Research and Practice, 17, 338-346.

Kirkland, K. y Kirkland, K. L. (2001). Frequency of Child Custody Evaluation Complaints and Related Disciplinary Action: A Survey of the Association of State and Provincial Psychology Boards. Professional Psychology: Research and Practice, 32 , 171-174.

Kumho Tire Company v. Carmichael, 526 U.S. 137 (1999).

LaFortune, K. A. y Carpenter, B. N. (1998). Custody evaluations: A survey of mental health profes- sionals. Behavioral Sciences and the Law, 16, 207-224.

Lally, S. J. (2001). Should human figure drawings be admitted into court? Journal of personality assessment, 76, 135-149.

Lampel, A. K. (1999). Use of the Millon Clinical Multiaxial Inventory-III in evaluating child custody litigants. American Journal of Forensic Psychology, 17, 19-31.

Leventhal, B., Kelman, J., Galatzer-Levy, R.M. y Kraus, L. (1999). Divorce, custody, and visitation in mid-childhood. En R.M.Galatzer-Levy y L. Kraus (Eds.), The scientific basis of child custody decisions (pp.205-225). New York: John Wiley and Son.

Ley de Enjuiciamiento Civil capitulo VI Sección $5^{\text {a }}$. Reforma de 2000 - vigente desde enero de 2001Libro II. Título I. Capítulo VI. Sección $5^{\text {a }}$ (artículos 335- 352).Retrieved 04/02/2008, 2008, from: http://noticias.juridicas.com/base_datos/Privado/l 1-2000.html

Lilienfeld, S. O., Wood, J. M. y Garb, H. N. (2000). The scientific status of projective

techniques. Psychological Science in the Public Interest, 1, 27-66.

Marquez, M.O., Vizcarro, C. Y FernándezBallesteros, R. (2004). Técnicas proyectivas. En R. Fernández-Ballesteros (Dir.), Evaluación psicológica. Conceptos, métodos y estudio de casos.(pp. 293-322) Madrid: Pirámide.

McCann, J. T., Flens, J. R., Campagna, V., Collman, P., Lazzaro, T., y Connor, E. (2001). The MCMIIII in child custody evaluations: A normative study. Journal of Forensic Psychology Practice, $1,27-44$.

Medoff, D. (2003). The scientific basis of psychological testing: Considerations following Daubert, Kumho, and Joiner. Family Court Review, 41, 199-213.

Meyer, D. J. (2006). Mala praxis en psiquiatría e investigaciones administrativas por alegación de mala conducta del médico. Psiquiatria forense. Clinicas psiquiátricas de Norteamerica, 29, 615628.

Millon, T. (2005). MCMI-II, Inventario Clínico Multiaxial de Millon II. Madrid: TEA Ediciones, S. A. 
Montero, J. (2001). Guarda y custodia de los hijos. Valencia: Tirant lo Blanch.

Montgomery, L. M., Cupit, B. E., y Wimberly, T. K. (1999). Complaints, malpractice, and management: Professional issues and personal experiences. Professional Psychology: Research and Practice, 30, 402-410.

Ortuño, P. (1998). Valoración Judicial de la intervención psicológica en procedimientos de familia. En Marrero, J.L. (coord.) Psicología Jurídica de la Familia. Madrid: Fundación UniversidadEmpresa, 287-313.

Otto, R. K. y Collins, R. (1994). Use of the MMPIUMMPI-A in child custody evaluations. En Y. S. Ben-Porath, J. R. Graham, G.C.N. Hall, R. D. Hirschman, y M. S. Zaragoza (Eds.), Forensic applications of the MMPI-2 (pp. 222-252). Beverly Hills, CA: Sage.

Otto, R. K., Edens, J. F. y Barcus, E.H. (2000). The use of psychological testing in child custody evaluations. Family and conciliation courts review, 38, 312-340.

Póstuma, A.B. y Harper, J.F. (1998). Comparison of MMPI-2 responses of child custody and personal injury litigants. Professional Psychology: research and practice, 29, 437-443.

Quinnell, F. A. y Bow, J. N. (2001). Psychological tests used in child custody evaluations, Behavioral Science and the Law, 19, 491-501.

Ramírez, M. (2006). El peritaje psicológico en el ámbito del derecho de familia. En J.C, Sierra, E. Jiménez y G. Buela-Casal (Comps.). Psicología Forense: Manual de técnicas y aplicaciones (pp. 223-245). Madrid: Biblioteca Nueva.

Rodríguez Sutil, C. (1999) Relación y diferencia entre el informe clínico y el informe forense. Papeles del psicologo,73. Recuperado el 30/03/ 2009 De: http://www.papelesdelpsicologo.es/vernumero. asp? $\mathrm{ID}=823$
Rodríguez Sutil, C. (2007) Evaluación de la personalidad y sus trastornos a través de los métodos proyectivos o pruebas basadas en la actuación (Perfomance-based). Clinica y salud: Revista de psicología clínica y de la salud, 18, 325-346.

Rogers, R., Salekin, R., y Sewell, K. (1999). Validation of the Millon Clinical Mutiaxial Inventory for axis II disorders: Does it meet the Daubert standard?. Law and Human Behavior, 23, 425-443.

Rorschach, H. (1962) Psychodiagnostik. Berna: Hans Huber Verlag.

Siegel, J. C. (1996). Traditional MMPI-2 validity indicators and initial presentation in custody evaluations.American Journal of Forensic Psychology, 14, 55-63.

Spielberger, C. D., Gorsuch, R.L., and Lushene. R.E. (1970). Manual for the State-Trait Anxiety Inventory. Palo Alto, CA: Consulting Psychologists Press.

Taylor, C.G., Norman, D.K., Murphy, J.M., Jellinek, M., Quinn, D., Poiterast, F.G., y Goshko, S.O. (1991). Diagnosed intellectual and emotional impairment among parents who seriously mistreat their children: prevalence, type and outcome in court sample. Child Abuse and Neglect, 15, 389401.

Tippins, T. M. yWittmann, J. P. (2005). Empirical and ethical problems with custody recommendations: A call for clinical humility and judicial vigilance. Family Court Review, 43, 193-218.

Vitacco, M. J. y Rogers, R. (2006). Psychopathy and response styles in sex offenders. In F. Saleh, A.Grudzinskas, y J. Bradford (Eds.), Sex offenders: Identification, risk assessment, treatment, and legal issues.New York: Oxford University Press.

Wechlser, D. (1955). Manual for the Wechsler Adult Intelligence Scale. New York: The Psychological Corp.

Artículo recibido: 14/11/2009

Revisión recibida: 01/12/2009

Artículo aceptado: 13/12/2009 